\title{
Balance político y económico de la Integración Andina durante 1980
}

INTRODUCGION

En el presente trabajo se realiza una descripción de la manifestación de las dimensiones políticas y económicas, del proceso de integración subregional andino durante 1980, para luego efectuar un balance de la interrelación entre las mismas.

Sin pretender abarcar todas las múltiples facetas de un proceso de tal complejidad, se describen los elementos de juicio más importantes de la dimensión política interna y externa, así como el grado de avance de los mecanismos básicos de la dimensión económica establecida por el Acuerdo de Cartagena.

Finalmente, se intenta realizar una evaluación de la interrelación entre ambas dimensiones y de los resultados de esa interrelación para la marcha del proceso de integración.

\section{A. LA DIMENSION POLITIGA}

\section{Reuniones de Jefes de Estado}

Durante 1980 se realizaron tres reuniones de presidentes y representantes personales de los presidentes de Colombia, del Ecuador, del Perú y de Venezuela, en las que se buscó impulsar el proceso de integración andino, a la vez que se hizo énfasis en la dimensión política del mismo.

La primera de ellas se llevó a cabo en Lima, el 29 de julio, en ocasión de la toma de posesión del cargo del Presidente del Perú, con la concurrencia de los mandatarios de Colombia y de Venezuela, de un representante personal del Presidente del Ecuador, y con la presencia del Presidente de Costa Rica, de un miembro de la Junta de Gobierno de Nicaragua, y del Presidente del Gobierno

* Nota: Las opiniones del autor son emitidas a títulos estrictamente personal $y$ no comprometen al organismo en que trabaja. 
de Espạña. En esta reunión se suscribió la "Declaración de Lima"1, en la que, entre otros temas, se abordan aspectos que hacen a la dimensión política interna y externa del proceso, así como a la asociación de la misma con el esquema de integración económica en el marco del Sistema Andino de integración.

En cuanto a la dimensión política interna, la "Declaración de Lima" expresa "el fortalecimiento de las instituciones democráticas constituye la mejor garantía para lograr un desarrollo integral y elevar la calidad de vida de los pueblos de la subregión", expresiones que a la vez de centrar la dimensión política interna en las formas democráticas de gobiemo, reflejan la intención de vincular las mismas con Jos objetivos y naturaleza del proceso de integración económica establecido en el Acuerdo de Cartagena, cuyos objetivos son "promover el desarrollo equilibrado y armónico... con la finalidad de procurar un mejoramiento persistente en el nivel de vida de los habitantes de la subregión" (art. 1).

Asimismo, la citada declaración señala: "Expresamos nuestra viva complacencia por la dimensión política y el perfeccionamiento del sistema jurídico-institucional del proceso de integración andi$\mathrm{no}^{2}$, experimentado a partir del Mandato de Cartagena ${ }^{3}$, como una consecuencia natural de la evolución favorable y la consolidación de la integración en el plano socioeconómico", lo que implica una evaluación de la integración económica caracterizada por su evolución favorable y su consolidación, a la vez que destaca la incorporación de la dimensión política al proceso a través del. Sistema Andino.

Esta última apreciación es explícitamente formulada en la declaración de referencia, al expresarse que: "Entendiendo el proceso como un fenómeno multidimensional, observamos que este desarrollo se ha manifestado sobre todo a través de la institucionalización del Consejo de Ministros de Relaciones Exteriores del Grupo Andino, del Parlamento Andino y del Tribunal de Justicia del Acuerdo de Cartagena", elementos incorporados al Sistema Andino durante 1979.

En relación con la dimensión política externa, la "Declaración de Lima" señala que las actividades desplegadas por los presidentes de los países miembros del Grupo Andino, del Consejo Andino

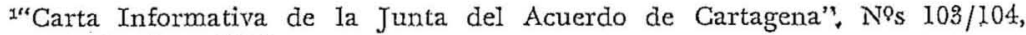
agosto-septiembre, 1980.

${ }^{2}$ Sobre el Sistema Andino véase "El proceso de integración subregional andino durante 1979", Luis De María, Integración Latinoamericana No 48, julio de 1980 .

${ }^{3}$ Suscrito por los Presidentes de Bolivia, Colombia, Ecuador, Perú y Venezuela en la ciudad de Cartagena, el 28 de marzo de 1979. 
y de los órganos principales del Sistema Andino, han reforzado el poder de negociación frente a terceros.

La segunda reunión a nivel presidencial se llevó a cabo el il de septiembre en Riobamba, con motivo del sesquicentenario de la aprobación de la Constitución de la República del Ecuador, ocasión en que los presidentes de Colombia, del Ecuador y de Venezuela, y el representante personal del Presidente del Perú, suscribieron una "Carta de Conducta", en la que, entre otros importantes aspectos, se afirma: "se ha alcanzado una etapa sustantiva del esquema: de integración económica que ha dado lugar a la formación de nuevos vínculos que han permitido a los países de la subregión lògrar niveles de concertación política ... la concertación de voluntades políticas contribuirá decisivamente a la afirmación... del sistema democrático y de los principios que lo sustentan".

Nuevamante, las más altas autoridades políticas de la subregión hacen referencia a que se ha alcanzado una etapa de consolidación del proceso en el plano económico, y que, como consecuencia de los vínculos establecidos en la dimensión económica, puede encararse la "concertación política", cuyo centro de gravedad es el sistema democrático, en la difícil situación de lograr esta concertación ante los hechos acaeciclos en Bolivia.

La última circunstancia señalada, hace suponer que el nivel de consolidación económica alcanzado por el proceso fue evaluado como lo suficientemente válido, no sólo como para permitir en forma general la concertación de voluntades políticas, sino como para soportar las tensiones generadas en el sistema ante la búsqueda de la concertación basada en las formas democráticas de gobierno. en las particulares circunstancias porque atravesaba Bolivia.

La tercera reunión de Jefes de Estado se realizó en Santa Marta cl 16 de diciembre, con motivo de cumplirse 150 años de la muerte del Libertador Simón Bolívar, con la concurrencia de los presidentes de Colombia, del Ecuador, del Perú y de Venezuela y de otros mandatarios de fuera de la subregión. Los presidentes suscribieron la "Declaración de Santa Marta" ", en la que reafirmaron la urgencia de acelerar el proceso de integración en que están comprometidos y señalaron: "La próxima entrada en vigor del Tribunal de Justicia, el ejercicio cle las funciones del Parlamento Andino, el ámbito atribuido al Consejo cle Ministros, $y$ todos aquellos otros entes especializados que forman el Sistema, deben fortificar al máximo la fe de los pueblos y gobiernos en las virtualida-

"Separata de "Carta Informativa de la Junta ciel Acuerdo de Cartagena", No 105 , octubre, 1980.

"Separata de la "Carta Informativa de la Junta del Acuerdo de Cartagena", No 107 , diciembre de 1980. 
Luis G. De Maria / Balance político y económico de la Integración...

des políticas y económicas de la integración", expresando: nuevamente la conjunción de las dimensiones política y económica en el Sistema Andino. Asimismo, expresaron que: "la institucionalización cle la democracia es la mejor garantía para la libertad y el desarrollo de sus pueblos", reiterando la asociación entre las formas democráticas de gobierno y el desarrollo.

\section{La proyección externa}

El principal componente del Sistema Andino en lo que hace a la proyección externa del mismo, es el Consejo de Ministros de Relaciones Exteriores (Consejo Andino), entre cuyas funciones cabe destacar la de formular la política exterior conjunta de los países de la subregión.

Dưrante 1980, se llevó a cabo en enero la primera reunión del Consejo, en Santa Cruz de la Sierra, Bolivia, suscribiéndose, entre otros acuerdos, uno respaldando las gestiones de la Comisión del Acuerdo de Cartagena para la colocación de hilados de algodón bolivianos en la Comunidad Económica Europea (GEE), y una declaración de solidaridad con Bolivia frente a la decisión de Estados 'Unidos de vender 35.000 toneladas de estaño de sus reservias estratégicas no comerciales ${ }^{6}$.

A continuación se realizó la segunda reunión del Consejo en Lima, Perú, en la que se suscribió un memorándum de entendimiento entre el Grupo Andino y el Brasil, constituréndose un mecanismo de intercambio de informaciones, consultas y coordinación entre ambos. A nivel político ese mecanismo estará constituido por reuniones entre el Consejo. Andino y el Canciller de Brasil, por comités sobre temas específicos, y por el comité mixto perma. nente formado por los embajadores de los países miembros del Grupo Andino acreditados en Brasilia, y por los representantes de Brasil que correspondan. Entre otros objetivos, este mecanismo tendrá los de promover las relaciones recíprocas, estimular esfuerzos de cooperación en diversas áreas, impulsar la cooperación e inte-. gración latinoamericanas, y estimular la convergencia de América Latina con los países en desarrollot.

En la tercera reunión del Consejo que se llevó a cabo en Quito, Ecuador, se firmó un acuerdo sometiendo a los Jefes de Estado de la subregión un proyecto de "Carta de Gonducta" (la cual fue suscrita por los presidentes en la citada reunión de Riobamba), en la que, entre otros enunciados políticos, se acordo propiciar una ma-

\footnotetext{
*Primera reunión del Consejo de Ministros de Relaciones Exteriores del Grupo: Andino, JuN/di 445 , 30 de enero de 1980.

"Segunda Reunión del Consejo Andino", Jun/di 456, il de abril de 1980.
} 
yor participación en las negociaciones de los problemas políticos y económicos que se debaten en la comunidad internacional, particularmente los que se refieren a la paz y seguridad y al Nuevo Orden Económico Internacional, en cooperación con los otros países latinoamericanos y del Tercer Mundo8.

Asimismo, durante la tercera reunión del Consejo, se llevó a cabo una reunión con el Canciller de la Argentina en la que se suscribió una declaración conjunta y un acta de entendimiento.

En la primera, ratificaron su apoyo al Sistema Económico Latinoamericano (SELA), reafirmaron su voluntad política para reestructurar la Asociación Latinoamericana de Libre Comercio (ALALC) y lograr así un nuevo orden de integración regional, consideraron conveniente iniciar el estudio y la negociación de un acuerdo de cooperación y desarrollo en el marco de la Comisión Mixta Andino-Argentina, y el Ganciller de la Argentina expresó el deseo de su país de adherir al convenio "Andrés Bello".

En el acta de entendimiento suscrita en esa oportunidad, se estableció un mecanismo de intercambio de informaciones, consultas y coordinación, compuesto a nivel político por reuniones entre el Consejo Andino y el Canciller de la Argentina, por comités o grupo de trabajo específicos y por el comité mixto permanente formado por los embajadores de los países de la subregión, acreditados en Buenos Aires y por representantes argentinos. Asimismo, en dicho acto se estableció que la identificación y desarrollo de actividades concretas de cooperación a nivel económico estarán a cargo de la Comisión Mixta Andino-Argentina.

Atendiendo a la invitación de la Comunidad Económica Europea (CEE), en mayo de este año se llevó a cabo un encuentro entre el Consejo Andino y los ministros de relaciones exteriores de los estados miembros de la CEE, en el que se suscribió una declaración conjunta, en la que reafimaron su.compromiso en favor de la cooperación entre los dos procesos de integración, y expresaron sus puntos de vista sobre diversos aspectos internacionales?.

En relación a los acuerdos logrados en la Ronda Tokyo del Acuerdo General de Aranceles y Comercio (GATT), los representantes de la CEE manifestaron su satisfacción respecto a los mismos, mientras que el Consejo Andino estimó que los resultados de dichas negociaciones no colmaron sus expectativas. En lo concerniente a los proyectos concretos para cuya ejecución la GEE brindaría su concurso financiero y técnico en la subregión andina. los representantes de la CEE destacaron su interés particular por los proyec-

"'Tercera Reunión del Consejo Andino", Juv/di 455, 19 de abril de 1980.

or Reunión del Consejo de Ministros de Relaciones Exteriores del Grupo Andino con los Ministros de Relaciones Exteriores de los Estados Miembros de las Comunidades Europeas" JuN/di 469, 14 de mayo de 1980. 
tos agxícolas, agroindustriales y rurales, y los miembros del Consejo Andino expresaron el deseo de que la cooperación financiera y técnica se extienda al campo industrial y de infraestructura.

Gabe señalar, que con el propósito de intensificar aún más las relaciones entre ambos esquemas de integración, durante 1980 se desarrollaron negociaciones orientadas a la suscripción de un convenio de cooperación, durante las cuales la cEE ha ido definiendo los linieamientos generales que orientarán su posición ${ }^{10}$. En ese sentido, la 'Comisión Ejecutiva de la Comunidad Económica Europea aprobó, en el primer trimestre del año, las propuestas para la iniciación de negociaciones con el Grupo Andino, las que posteriormente fueron debatidas en los órganos principales de la CEE.

Entre los lineamientos generales aprobados por la Comisión de la CEE, cabe destacar que se propone un cuadro de colaboración que no sea preferencial, que tenga carácter evolutivo y que no excluya a priori ningún sector de competencia comunitaria, y que tenga en cuenta las diferencias en el desarrollo relativo de las partes. Asimismo, la propuesta de la CEE se orienta a un convenio que comporte la aplicación de la cláusula de la nación más favorecida, que tienda a intensificar y diversificar las relaciones comerciales entre las dos regiones, y que siente las bases para una cooperación económica en los sectores que las partes deseen.

Durante 1980, se realizaron frecuentes contactos entre representantes de ambas partes, entre los que cabe destacar la citada reunión entre el Consejo Andino y los Ministros de Relaciones Exteriores de los países de la CEE, y las visitas a la sede de la Junta del Acuerdo de Cartagena, realizadas por el responsable en la Comisión de la CEE de las relaciones externas, y del Ministro de Relaciones Exteriores de Gran Bretaña. Como culminación de este proceso de definiciones preliminares, en junio de 1980 se inició en Bruselas, la primera ronda de negociaciones entre ambas partes, con la participación de los miembros de la Junta del Acuerdo de Cartagena por un lado, y de los miembros de la Comisión de la CEE, por el otrol1.

Asimismo, en mayo de este año se llevó en Madrid una reunión entre el Consejo Andino y el Ministro de Asuntos Exteriores de España, ocasión en la que suscribieron una declaración conjunta. Con respecto a las negociaciones a efectuarse entre el Grupo Andino y la CEE, España declaró su complacencia por esta aproximación entre las dos organizaciones regionales, y ambas partes concor-daron en la conveniencia de examinar las posibilidades de concer-

${ }^{10} \mathrm{Los}$ países andinos definieron los elementos generales de su posición en 1979. Véase "El proceso de Integración en América Latina durante 1979", INTAI, agosto de 1980.

11" Carta Informativa de la Junta del Acuerdo de Cartagena", No 101, junio de 1980. 
tación, en temas de interés común sobre sus respectivas relaciones con los órganos competentes de la cEE. Entre otros asuntos tratados en dicha reunión, el Ministro de Asuntos Exteriores de España expresó formalmente el deseo de su país de adherirse al Convenio "Andrés Bello"12.

Por otra parte, en octubre de 1980, se llevó a cabo en Washington la primera reunión del grupo especial de comercio entre el Grupo Andino y los Estados Unidos de América ${ }^{13}$. En esta reunión se abordaron Ios siguientes temas: el sistema generalizado de preferencias de los Estados Unidos de América, las negociaciones comerciales multilaterales del GATT, medidas para mejorar las relaciones comerciales entre ambas partes, y aspectos vinculados con los productos básicos.

Con respecto al sistema generalizado de preferencias, las conversaciones se desarrollaron en torno a los productos especificos y a las reglamentaciones de este sistema, acordándose pautas y procedimientos que facilitarán la presentación conjunta de productos de interés para la subregión. Asimismo, durante la reunión se presentó formalmente la solicitud de que el Grupo Andino sea considerado como una asociación regional para efectos del origen acumulado.

De otro lado, en el primer encuentro subregional de Ciencia y Tecnología celebrado en Lima en abril de 1980, la Junta del Acuerdo de Cartagena, recibió observaciones y sugerencias sobre las acciones específicas a seguir en las negociaciones con los Estados Unidos de América en el área de ciencia y tecnología, para la cual también se ha previsto la constitución de un grupo especial.

Por último, entre los elementos más importantes de la proyección externa del Grupo Andino cabe destacar la participación coniunta de sus países miembros en el proceso de reestructuración de la ALALc. Además de la posición negociadora conjunta, el modelo económico e institucional andino se ha proyectado en la nueva estructura de ALADr, en los acuerdos de complementación económica y en el robustecimiento del rol de la Secretaría de la Asociación ${ }^{15}$.

Por su parte, los presidentes de los países andinos, en oportuni-

1arcReunión del Consejo de Ministros de Relaciones Exteriores del Grupo Andino con el Ministro de Asuntos Exteriores de España", Jun/di 471, 21 de mayo de 1980.

${ }^{13 E n}$ noviembre de 1979 se suscribió un memorándum de entendimiento entre la Comisión del Acuerdo de Cartagena y el Gobierno de los Estados Unidos de América, cstableciendo el interés de ambas partes por desarrollar una cooperación efectiva en las áreas de comercio, financiamiento, ciencia y tecnología, y desarrollo industrial, agrícola $y$ de infraestructura, incluyendo transporte. Asimismo, acordaron constituir grupos especiales para cada una de dichas áreas, con miras a firmar un acuerdo de cooperación económica en base a sus resultados.

${ }^{7 \sigma}$ Sobre las nuevas caracteristicas de la Secretaría de la Asociación Latino- 
Luis G. De María / Balance político y económico de la Integración...

dad de suscribir la citada "Declaración de Lima" expresaron, en relación a la Asociación Latinoamericana de Integración (ALADI) : "Brindamos nuestro apoyo político a este nuevo esquema amplio de integración regional que sustituye un modelo tradicional que no se adecuaba a la realidad latinoamericana y a los principios de equidad $y$ desarrollo armónico. Estimamos como imprescindible que nuestros países mantengan una participación activa y coordinada en este nuevo esquema de integración regional, en forma compatible con la consolidación del Grupo Andino".

\section{B. LA DIMENSION ECONOMICA}

\section{La formación del mercado ampliado}

El proceso de formación del mexcado ampilado en el Grupo Andıno se realiza a través de la implementación de dos mecanismos básicos: el programa de liberación y el Arancel Externo Común (AEC) .

En cuanto al primer mecanismo mencionado, en 1979 se habia reducido el nivel arancelario interno inicial para Lolombia, feru y venezuela en un 74\%, correspondiendo una disminución automática adicional del $6 \%$ el 31 de diciembre de 1980. En los próximos tres años a través de rebajas del $6 \%$ del nivel arancelario interno en 1981 y en 1982, y de una última rebaja del $8 \%$ el 31 de diciembre de 1983, se completaría el programa de liberación para los tres países de mayor desarrollo relativo de la subregión.

Respecto a Bolivia y el Ecuador, que gozan ya del mercado ampliado, deben iniciar el cumplimiento del programa de liberación el 31 de diciembre de 1980, comenzando su reducción arancelaria en tavor de los restantes miembros el 31 de diciembre de 1981, hasta la liberación plena de aranceles internos en 1990, según lo establecido en el artículo 100 del Acuerdo de Cartagena. Cabe señalar al respecto, que en base a evaluaciones realizadas por la Junta, sobre los resultados que obtengan Bolivia y el Ecuador en su intercambio con los demás países miembros, y en la medida en que estén aprovechando los beneficios del mercado ampliado, la Comisión del Acuerdo de Cartagena podrá revisar los plazos señalados en el párrafo precedente (Artículo 10l del Acuerdo de Cartagena).

De lo expuesto, surge que el programa de liberación se encuentra en una fase avanzada, cuya próxima etapa sería la iniciación del mismo por parte de los paises de menor desarrollo relativo a

americana de Integración (ALADI), véase "Análisis comparativo de los Tratados de Montevideo 1960 y 1980", Raymundo Barros Charlín, Integración Latinoamericana $N^{\circ} 50$. 
la subregión, de no mediar una evaluación negativa sobre los beneficios que obtienen del proceso de integración económico dichos países.

En cuanto al Arancel Externo Común (AEC), si bien se avanzó durante el año en la definición y precisión tanto en materia de niveles de protección máxima y mínima así como en el procedịmiento de aproximación al AEG, no se pudo lograr en 1980, la decisión necesaria para superar la situación de incumplimiento en el plano formal, y de indefinición en materia económica.

Los plazos establecidos en el Acuerdo de Cartagena para la aprobación del AEG (31 de diciembre a más tardar) y para que Colombia, Perú y Venezuela comenzaran el proceso de aproximación al mismo (31 de diciembre de 1980), no se han podido cumplir.

Pese a ello, vale la pena destacar los esfuerzos realizados por el órgano técnico comunitario en la precisión de la propuesta dél AEa. En efecto, siguiendo los lineamientos básicos presentados en el xxy Período de Sesiones Ordinarias de la Comisión ${ }^{16}$, la Junta presentó una propuesta modificada sobre el AEG y la armonización de los instrumentos de comercio exterior ${ }^{17}$, consistente en niveles de gravámenes máximos y mínimos de protección a la producción subregional.

El AEG al que se llegaría en 1990, en caso de aprobarse la citada propuesta, estaría conformado por una franja de protección, cuyo nivel mínimo tendría un rango de protección efectiva ${ }^{18}$ de 0 a 70 puntos, y los incentivos a las diferentes actividades ductivas surgen como consecuencia de la aplicación de los llamados criterios básicos y correctivos. Como criterios básicos ${ }^{1.9}$ se consideraron: la generación de empleo, la contribución tecnológica y el nivel de incipiencia industrial, a los que se agregan los siguientes criterios correctivos: ${ }^{20}$ propensión a importar, insumos agropecuarios, productos de exportación tradicional, productos de interés social y productos no producibles.

El nivel mínimo promedio de protección nominal del AEG sería

28"Bases para la elaboración de una propuesta modificada sobre arancel externo común y armonización de los instrumentos de comercio exterior", com/ XXY-E/at 2, 29 de febrero de 1980.

17"Propuesta de la Junta sobre Arancel Externo Común y armonización de los instrumentos de comercio exterior", Jun/Propuesta 96/Mod. 1, 17 de mayo de 1980.

${ }^{18} \mathrm{La}$ protección efectiva no sólo toma en cuenta la protección al producto final, sino también la protección de los insumos, midiendo la relación entre el valor agregado a los precios internos con el valor agregado a precios internacionales.

19Véase "El proceso de integración subregional andino durante 1979", op. cit.

vóase: "Análisis de criterios correctivos del Arancel Externo Común de la Propuesta 96/Mod. 1", JuN/di 517, 27 de noviembre de 1980. 
Luis G. De Maria / Balance político y económico de Ia Integración...

de $25 \%$, con una desviación estándard de 10 puntos $^{21}$, y el nivel de protección máxima nominal se determinaría como dos veces y media el nivel de los gravámenes mínimos, siempre que el gravamen resultante no supere el valor de $100 \%$ ad valorem cIF.

En cuanto al comercio intrarregional, las exportaciones intrasubregionales en 1980, se estiman en 1.400 millones de dólares, lo que representa un $4,3 \%$ de las exportaciones de la subregión al mundo. Este coeficiente de participación del comercio intrasubregional en las exportaciones totales de la subregión, si bien es superior al que existía al comienzo del proceso de integración andino, es aún bajo, y no implica que se haya logrado una etapa sustantiva en el proceso de integración económica.

Sin embargo, casi el $80 \%$ de las exportaciones intrasubregionales está constituido por productos no tradicionales, lo que refleja la importancia cualitativa $y$ potencial del esquema de integración económica andino, si se tiene en cuenta las posibilidades que brinda para la diversificación del comercio exterior de los países de la subregión y para el comercio de manufacturas.

EXPORTACIONES DEL GRUPO ANDINO EN 1980

(Millones de dólares)

\begin{tabular}{|c|c|c|c|c|}
\hline Pats & $\begin{array}{c}\text { Exportaciones } \\
\text { intrasub- } \\
\text { regionales } \\
\text { (1) }\end{array}$ & $\begin{array}{l}\text { Participación en } \\
\text { el comercio intra- } \\
\text { subregional }\end{array}$ & $\begin{array}{l}\text { Exportaciones } \\
\text { totales } \\
\text { (2) }\end{array}$ & $\frac{(1)}{(2)} \times 100$ \\
\hline Bolivia & 23 & $1,7 \%$ & 910 & $2,5 \%$ \\
\hline Colombia & 457 & $32,2 \%$ & 4.600 & $9,9 \%$ \\
\hline Ecuador & 118 & $8,4 \%$ & 2.590 & $4,6 \%$ \\
\hline Perú & 543 & $38,3 \%$ & 3.900 & $13,9 \%$ \\
\hline Venezuela & 275 & $19,4 \%$ & 20.600 & $1,3 \%$ \\
\hline Subregión & 1.416 & 100 & 32.600 & $4,3 \%$ \\
\hline
\end{tabular}

Fuentes: "Noticias para la Prensa", Junac, No 144, y "Notas sobre la economía y el desarrollo de América", GEPAL, No 333.

En cuanto a la participación de los países miembros en el comercio intrasubregional, Colombia, el Perú y Venezuela representaron en conjunto el 90\%, mientras que Bolivia y el Ecuador sólo el $10 \%$, mostrando un fuerte grado de concentración en las exportaciones de los países de mayor desarrollo relativo. A su vez, las ex-

2a "Información estadística sobre los niveles mínimos de la franja del Arancel Externo Común para 1983 y 1990, y aranceles nacionales vigentes", com/ $\mathrm{xxy}$ E/dt 4,5 de marzo de 1980. 
portaciones a la subregión de Bolivia representaron sólo el $2 \%$ del comercio intrasubregional. Dado que entre los objetivos del desarrollo equilibrado y armónico establecido en el Acuerdo de Cartagena, figura en primer término la equitatividad en la distribución de los beneficios del proceso, las citras mencionadas sugieren que los objetivos del mismo aún no se han consolidado en el actual estado del esquema de integración económica andino, y particularmente con respecto a Bolivia.

\section{Programación Industrial}

Si bien el Acuerdo de Gartagena establece la posibilidad de encarar permanentemente la programación inclustrial, se buscó inicialmente concentrar la misma en un conjunto de sectores prioritarios, para lo cual se establecieron plazos tanto para determinar una nómina de productos reservados para programas industriales, como para la aprobación de los programas respectivos.

En base a la nómina de reserva establecida por la Decisión 25, la Junta elaboró propuestas sobre 8 sectores industriales: metalmecánico, petroquímico, automotriz, siderúrgico, fertilizantes, electrónico y telecomunicaciones, químico, y tarmoquímico, habiéndose aprobado hasta comienzos de 1980 programas sectoriales de desarro110 industrial en los tres primeros sectores a través de las decisiones 57 y 146,91 y 120 respectivamente

Yor otra parte, la Decisión 143, aprobada el año pasado, prorrogó el término del período de reserva para la programación indusurial hasta el 31 de diciembre de 1980.

De las 5 propuestas de la Junta que fueron analizadas durante el año, correspondientes a los sectores siderúrgico (prop. 66), fertilizantes (pro. 47), electrónico y telecomunicaciones (prop. 69), químico (prop. 91) y farmoquímico (prop. 92), sólo se produjeron decisiones en el sector siderúrgico y en el de fertilizantes.

En el xxxi período de sesiones ordinarias de la Comisión, se aprobó la Decisión 160 sobre el Programa Sectorial de Desarrollo de la Industria Siderúrgica, conteniendo exclusivamente el conjunto de productos objeto del programa; los restantes instrumentos del programa serán aprobados por la Comisión, a propuesta de la Junta, antes del 31 de diciembre de 1981. Mientras no se aprueben los restantes instrumentos del programa, los países miembros podrán mantener en sus listas de excepciones los productos objeto del programa que estuviesen incluidos en las mismas.

En cuanto al sector industrial de fertilizantes, la Decisión 162,

פVéase "Informe de la Junta sobre la elaboración de programas sectoriales e intersectoriales de desarrollo industrial". JuN/dt 127, 17 de enero de 1980. 
aprobada en el xxvix periodo de sesiones extraordinarias de la Comisión, define el programa de liberación y el arancel externo común para los productos del sector, en atención a que, vencido el período de reserva, no fueron incluidos en programas sectoriales de desarrollo industrial, y aprueba la nómina de productos del sector que no se producen en la subregión.

De este modo, al finalizar el año, venció el plazo para la aprobación de programas sectoriales de desarrollo industrial, habiéndose establecido que durante $198 \mathrm{I}$ se completará la definición del programa siderúrgico.

De los tres programas aprobados, el programa de la industria automotriz es el que había despertado mayores expectativas en cuanto a la importancia del impacto de su implementación en las economías de los países miembros. Durante 1980, se aprobaron 3 decisiones sobre este programa, modificando los plazos límites para la definición de los modelos básicos del mismo23. A principios de 1980, el plazo límite para su definición era el 31 de diciembre de 1979, establecido por la Decisión 149 del xxiv período de sesiones extraordinarias de la Comisión, sin que se hubiere podido cum$\mathrm{plir}^{24}$.

En marzo de 1980, clurante el xxvI período de sesiones extraordinarias de la Comisión, se aprobó la Decisión 152, que prorroga el plazo para la definición de los modelos básicos hasta el 30 de abril de 1980, en las categorías A3, A4, B2.1, B3, B4 y G que se habían asignado a Colombia, al Perú y a Venezuela. Asimismo, en el xxrx período de sesiones ordinarias de la Comisión, aprobó la Decisión 158, que amplió hasta el 15 de julio de 1980 el plazo para la definición de los modelos básicos de dichas categorias.

Posteriormente, en el xxvII periodo de sesiones extraordinarias de la Comisión se aprobó la Decisión 159, en la que nuevamente se amplían los plazos para la definición de los modelos básicos hasta el 31 de agosto de 1980 para las categorías A3 y A4 y para las categorías B2.1, B3, B4 y C hasta el 15 de octubre de 1980. Al finalizar el año, aún no se había completado la definición de los modelos básicos.

Cabe señalar que durante la reunión del Consejo Directivo de la Asociación de Cámaras de Fabricantes de Productos Automotores del Grupo Andino (GAFAndina), realizada en Lima a principios

${ }^{23} \mathrm{E} 1$ modelo básico es aquel que cada país elige dentro de cada una de las categorías que le han sido asignadas. La definición se hace describiendo las caracterfsticas técnicas de los siguientes componentes fundamentales: motor, cajas de velocidades mecánica, caja de velocidades automática, eje o ejes tractores, eje portante, caja de dirección mecánica, y caja de dirección hidráulica.

${ }^{a} \mathrm{E} 1$ plazo original establecido por el artículo 86 de la Decisión 120 , para la definición de los modelos básicos era el 31 de diciembre de 1978. 
de 1980 , se reiteró el pleno apoyo a la Decisión 120 y se solicitó a los gobiernos de los países miembros "la puesta en vigencia de la Decisión 120 no solamente a nivel jurfdico, sino fundamentalmente a nivel operativo, y el cumplimiento de los plazos estipulaclos para la definición de los modelos básicos de vehículos"'25.

Entre las causas posibles del retraso en la definición de los modelos básicos, pueden señalarse tanto una cierta disminución del interés coyuntural en algún país miembro, por un programa basado en altos aranceles comunes; como la incertidumbre que crean los cambios tecnológicos que se producen en la industria automotriz a nivel mundial y que pueden cuestionar la validez de algunas asignaciones.

En cuanto al programa petroquímico, durante 1980 se han realizado estudios tendientes a actualizar este programa sectorial de desarrollo industrial ${ }^{20}$.

La Comisión, en su vigésimo período de sesiones ordinarias, tomó conocimiento de clichos estudios y resolvió constituir un grupo de trabajo ${ }^{27}$, con el fin de analizar las pautas para el reajuste de la Decisión 91.

En base al informe de dicho grupo de trabajo, y a las consideraciones de la Comisión, la Junta elaboró la Propuesta 1192s, conteniendo los ajustes necesarios al programa petroquímico.

En la propuesta de ajuste se retiran la relación de productos asignados a 'Chile, se incorpora el nuevo item correspondiente a poliéteres polioles derivados del óxido de propileno asignado a Bolivia y Venezuela, y se prorrogan diversas fechas determinadas por la Decisión 91, estableciendo un nuevo cronograma de plazos para el cumplimiento del programa. Asimismo se incorpora la posibilidad, por parte de los países miembros, de aplicar medidas de fomento a las exportaciones a la subregión, y se establece el monto máximo de las transferencias, abonos o reintegros, un proceso gradual para su aplicación, un tratamiento preferencial en los mismos para Bolivia $y$ el Ecuador y un tratamiento preferencial adicional para Bolivia.

En relación al programa metalmecánico, la Decisión 146 aprobada en 1979 sobre la reestructuración del programa metalmecánico e incorporación de Venezuela al mismo, establece en el artículo 33 que el Anexo xil conteniendo las asignaciones con producción

25"Boletín Automotor", No 2, octubre de 1980, Junac.

20"Informe de la Junta sobre el Programa Petroquímico y los Avances respecto del Ajuste de la Decisión 91", com xxix/ct 2\%, 17 de abril cle 1980.

sf"Informe del Grupo de Trabajo de la Comisión sobre el punto 7 de la agenda-Programa Petroquímico". CoM/xxu/dt 17, 31 de mayo de 1980.

ss"Propuesta de la Junta sobre el ajuste al programa sectorial de desarrollo de la industria petroquímica". JuN/Propuesta 119, 24 de junio de 1980.

$$
\left[\begin{array}{llll}
4 & 0 & 6
\end{array}\right]
$$


verificada, deberá ser actualizado luego que se verifique la producción existente en Venezuela a la fecha de aprobación de dicha Decisión. En base a la Resolución 141, que notificó a los países la existencia de producción en la subregión, la Junta elaboró la Propuesta $116^{29}$, para la actualización de las asignaciones con producción verificada del programa metalmecánico.

La Propuesta 116, analizada durante el vigesimonoveno periodo de sesiones ordinarias de la Comisión, dio origen a la Decisión 156, aprobada por la Comisión en ese período de sesiones, actualizando el Anexo xII de la Decisión 146. Dicho Anexo actualizado incorpora los cambios necesarios a las verificaciones de producción correspondientes a la aplicación de la Decisión 57, debido a las nuevas condiciones derivadas de la Decisión 146; así, para la unidad 6 (maquinaria de molinería), se considera que incluye sólo los. molinos de harina panificable, equipos cuya producción aún no se ha iniciado en la subregión, y para la unidad 35.14 (taxímetros y parquímetros), la producción no ha sido verificada en el nuevo país miembro asignatario.

En cuanto al programa siderúrgico, a principios de año se llevó a cabo la rv Reunión de Expertos Gubernamentales de la Industria Siderúrgica ${ }^{30}$, en la que se analizaron las características básicas propuestas por la Junta para la programación del sector. Entre otros importantes aspectos tratados, cabe destacar que sobre las empresas multinacionales propuestas como instrumentos del programa, Venezuela señaló que debían ser redefinidas como empresas con fines de lucro a fin de que puedan autofinanciarse, Colombia señaló que las empresas no deberían ser constituidas por los. gobiernos sino por las empresas siderúrgicas de los páses y el Ecuador manifestó que su creación debe responder al acuerdo de las empresas siderúrgicas nacionales y no como obligación gubernamental.

En el xxIx período de sesiones ordinarias de la Comisión se analizó la Propuesta 66/Mod. I de la Junta ${ }^{31}$, y en noviembre de este año se llevó a cabo la primera reunión del Grupo de Alto Nivel de la Comisión para examinar dicha propuesta ${ }^{\mathbf{3 2}}$.

Posteriormente, la Junta elaboró una zuueva versión ${ }^{38}$ del pro-

\footnotetext{
296، Propuesta de la Junta para la actualización del Anexo xil de la Decisión 146". JUN/Propuesta 116, 23 de abril de 1980.

"Acta Final de la Cuarta Reunión de expertos gubernamentales de la industria siderúrgica". JUN/REg. 15/IV/Acta Final, 28 de febrero de 1980.

at"Contenido y descripción de la propuesta de la Junta sobre el programa

de desarrollo de la industria siderúrgica", com/ xxrx/dt II, 23 de mayo de 1980.

as"Acta Final de la primera reunión del grupo de Alto Nivel de la Comisión para examinar la Propuesta 66/Mod. 1 de la Junta", Com/GAN. Is/I Acta
Final, 7 de noviembre de 1980 .
}

sa"Propuesta de la Junta sobre el programa sectorial de desarrollo de la 
grama siderúrgico, en la que se establecen como objetivos específicos del mismo:

a) promover el desarrollo de lia industria siderúrgica en cada uno de sos países miembros.

b) abastecer los requerimientos de productos de acero, con suministros de origen subregional, preferentemente.

c) racionalizar las producciones siderúrgicas de los países miembros, para obtener un desarrollo acelerado, eficiente y armónico del sector.

d) estımular el establecimiento de la industria siderúrgica en Bolivia y el Ecuador.

e) utilizar racionalmente los recursos existentes en la subregión, particularmente aquellos no renovables.

f) establecer corrientes de comercio de los productos del sector y sus insumos entre los países miembros.

g) promover la exportación de productos subregionales hacia terceros mercados.

h) promover el uso de la capacidad subregional de ingenieríi, consultoría, tabricación de equipos e investigación tecnologica.

i) estimular la modernización de las plantas existentes, cuando ello sea necesario, adaptándolas a las nuevas tecnologías siderúrgicas.

Asimismo, la propuesta de la Junta contiene los siguientes mecanismos del programa: programa de liberación, arancel externo común, normas de origen, armonización de polítıcas y acciones especiticas en beneficio de Bolivia y Ecuador. Entre otros compromisos del programa, corresponde a la Junta elaborar un Sistema de Información de Precios del sector para productos semiterminados, que comprendería precios de los mercados internos, de exportación y de terceros países abastecedores de la subregión; y a los países miembros corresponde: proporcionar información sobre los programas nacionales de producción, estimación de demandas internas y de los faltantes y excedentes de producción, coordinar sus planes de inversión, con el fin de promover acciones de racionali. zación, promover preferentemente el abastecimiento con los pro. ductos de la subregión, y emprender una normalización técnica con los productos del programa.

Cabe señalar que en la última versión de la propuesta de la Junta para el sector siderúrgico (pro. 66/Mod. 2) no se hace referencia explícita a las empresas multinacionales contempladas inicialmente en el documento de bases para el sector ${ }^{34}$.

industria siderúrgica". JuN/Propuesta 66/Mod. 2, 27 de noviembre de 1980.

"Véase "Bases para la propuesta de la Junta sobre el desarrollo de la industria siderúrgica". JuN/dt 120, 30 de octubre de 1979. 
Por último, durante el $x \times x i$ período de sesiones ordinarias de la Comisión, se aprobó la Decisión 160 sobre el Programa Sectorial de Desarrollo de la Industria Siderúrgica, que establece el conjunto de productos objeto del programa. Asimismo, establece que los restantes instrumentos clel programa serán aprobados por la Comisión, a propuesta de la Junta, conforme a lo dispuesto en el capítulo iv del Acuerdo, antes del 31 de diciembre de 1981.

De lo expuesto surge que la programación industrial en el Grupo Andino presenta un lento avance, y aún está en la etapa de consoliclación efectiva, sin que sus efectos potenciales, y particularmente el tratamiento preferencial a los países de menor desarrollo relativo, se hayan plasmado aún en vínculos económicos plenamente efectivos.

\section{G. BALANCE DEL AÑO}

La dimensión política durante 1980, presentó resultados claramente diferenciados en sus planos externo e interno. En el primero, como se expuso en la sección pertinente, el Grupo Andino manifestó una presencia activa y positiva en cuanto al incremento de su poder de negociación frente a terceros países y agrupaciones de países, clestacándose las negociaciones en el seno de la Asociación Latinoamericana de Libre Comercio y con los países mayores de la región, el comienzo de lass negociaciones con la $\mathrm{CEE}$, y la reunión del grupo especial de comercio en el marco del memorándum de entendimiento con los Estados Unidos de América.

En contraste, el enfoque dado a la dimensión política interna, presentó resultados clesfavorables para el proceso de integración andino. Ello se puso de manifiesto en la controversia suscitada con Bolivia en torno a la naturaleza y objetivos del proceso, ya que, ante los hechos descritos en la primera sección del presente trabajo, la Cancillería de Bolivia emitió una declaración, el 18 de diciembre, señalando que: "en vista de la interpretación ideológica que pretenden dar al Acuerdo algunos de sus miembros, desvirtuando sus objetivos esenciales... declara la abstención de su participación activa, sin que esto signifique un apartamiento definitivo del Tratado ni la renuncia a sus objetivos específicos".

Como consecuencia de rechazar la asociación entre las formas democráticas de gobierno y los objetivos económicos del Acuerdo de Cartagena, y dado que, como se señaló anteriormente, esta asociación estuvo materializada en la incorporación de la dimensión política interna a través del Sistema Andino, también se llegó al cuestionamiento implícito, de la validez de las instituciones polí. ticas del mismo.

En ese sentido, el 19 de diciembre, la Secretaría General de In- 
tegración de Bolivia, emitió un comunicado de prensa señalando: "El proceso de integración andino es económico y social, estando delimitados sus objetivos y mecanismos en el Acuerdo de Cartagena y sus Protocolos modificatorios... De ahí que el Gobierno de Bolivia reconozca como únicos órganos válidos a la Comisión, a la Junta, y al Tribunal Andino de Justicia... Por existir en ese caso una controversia que requiere una interpretación de carácter jurídico de los objetivos básicos del Acuerclo y los procedimientos para alcanzarlos, el Gobierno de Bolivia planteará el caso ante... el tribunal arbitral para la solución de controversias de la Asociación Latinoamericana de Libre Comercio... ${ }^{35}$. Entre tanto este tribunal no se pronuncie sobre la correcta interpretación de la controversia que se ha suscitado, Bolivia se abstendrá de participar en la Comisión del Acuerdo de Cartagena".

Es indudable que la búsqueda de "concertación de voluntades políticas" en torno de las formas democráticas de gobierno, en las circunstancias particulares por las que atravesaba Bolivia, no constituyó un apoyo político al proceso de integración económico y social, sino que, por el contrario, creó presiones que debieron ser absorbidas por el grado de consolidación del esquema de integración económica.

Por otra parte, lo expuesto en la sección correspondiente a la dimensión económica del proceso, sugiere que se estaba en proceso de alcanzar una etapa sustantiva en el esquema de integración económica, pero que la misma aún no se había alcanzado. Ello es así, porque para que la integración económica pueda caracterizarse como consolidada, debiera, como mínimo haberse aprobado e implementado el Arancel Externo Común, y la programación industrial debiera estar en pleno funcionamiento efectivo, lo que daría como resultado, niveles de participación del comercio intrasubregional en el comercio total muy superiores a los registrados en 1980.

Al respecto, cabe señalar la evaluación hecha por el órgano técnico comunitario del Acuerclo de Gartagena en mayo de 1979: "Podría decirse que el lapso que media entre la próxima reunión de Presidentes Andinos que tendrá lugar el 26 de mayo de este año y la convocada para diciembre de 1980 para conmemorar el

${ }^{3}$ El mencionacto Tribunal o Protocolo para la Solución de Controversias pertenecen al ordenamiento jurídico de ALacc. A juicio de $R$. Barros Charlín "queda en claro que el Actuerdo de Cartagena no es uno de los acuerdos previstos en el Tratado de Montevideo 1980, y un ordenamiento jurídico cobra plena autonomía en relación con la ALADI, tal como lo dispone el Protocolo que crea el Tribunal de Justicia del Acuerdo de Cartagena y el artículo 44 del Tratado de Montevideo 1980", ver Revista Integración Latinoamericana No 50, p. 42. 
Luis G. De Maria / Balance político y económico de la Integración...

Sesquicentenario de la muerte clel Libertador, que a su vez coincide con el vencimiento de los plazos principales fijados en el Protocolo de Arequipa, el Grupo Andino vivirá la última etapa de su proceso de formación, luego de la cual ingresará a la primera de su periodo de madurez" 3 .

Las consideraciones precedentes permiten concluir que la evaluación realizada a nivel político sobre el avance del proceso de integración económica es incorrecto en dos sentidos: a) el esquema de integración económica aún no se había consolidado, y b) el mismo no permitía absorber las presiones generadas por una concertación política interna en torno a las formas democráticas de gobierno.

Esta inversión en los términos correctos de interrelación entre las dimensiones política y económica puede incluso afectar, de persistir, la consolidación del esquema de integración económica, y de este modo repercutir desfavorablemente en la proyección externa de la subregión.

El balance esbozado, sugiere la necesidad imperiosa de que la dimensión política interna se caracterice por el apoyo decidido a la consolidación del esquema económico, sin pretender que el mismo pueda permitir, hasta que efectivamente se produzcan vínculos económicos sustantivos, absorber presiones de una concertación politica conflictiva.

${ }^{36 s}$ Evaluación del Proceso de Integración 1969-1979", Junta del Acuerdo de Gartagena;, mayo de 1979. 